\title{
Hemispace-visual field interactions in visual extinction
}

\author{
STEVEN Z RAPCSAK, ROBERT T WATSON, KENNETH M HEILMAN
}

From the Department of Neurology, College of Medicine, University of Florida, and Veterans Administration Medical Center, Gainesville, Florida, USA

SUMMARY Visual extinction was studied in a patient with neglect from a right hemispheric lesion. Extinction occurred during double simultaneous stimulation within the same visual hemi-field (VHF). This finding suggests that interhemispheric rivalry is not a critical factor. During double simultaneous stimulation within the same VHF as well as with stimuli in different VHFs, the severity of extinction was determined by both the retinotopic and the hemispatial position of the extinguished stimulus. The location of the other stimulus, however, did not seem relevant. It is proposed that damage to a corticolimbic-reticular system reduces attentional capacity and that extinction during double simultaneous stimulation in the visual modality reflects an inability to distribute the limited attentional resources equally to two different locations. The severity of extinction is a measure of an unequal distribution of attention along a gradient within visual space.

Sensory extinction can be defined as a failure to report or respond to one of two simultaneously presented stimuli, despite the fact that each stimulus is correctly detected and localised when presented in isolation. Extinction most often occurs after damage to the cerebral hemispheres, and although left sided extinction had been reported to follow left hemispheric lesions, ${ }^{1}$ it is usually the stimulus presented on the side opposite the lesion that is extinguished when bilateral simultaneous stimulation is given. Extinction may be demonstrated in one modality only, or it may be multimodal.

Although several hypotheses had been advanced to explain extinction, its neuronal mechanisms remain poorly understood. Nathan ${ }^{2}$ and Reider $^{3}$ proposed that extinction during bilateral simultaneous stimulation resulted from suppression of the damaged hemisphere by the intact one. Along similar lines, Furmanski ${ }^{4}$ in his model emphasised interactions between ipsilateral and contralateral cortical "suppressor" areas and thalamic relay nuclei. To explain extinction by the nucleus reticularis thalami model, Heilman et al $^{5}$ posited that each association cortex may project to both ipsilateral and contralateral thalamic reticular nuclei. Whereas the ipsilateral

\footnotetext{
Address for reprint requests: Kenneth M Heilman, M.D., Box J-236, Department of Neurology, College of Medicine, University of Florida, Gainesville, FL 32610, USA.

Received 5 August 1986 and in revised form 4 February 1987. Accepted 11 February 1987
}

projections from association cortex may inhibit the nucleus reticularis thalami, the contralateral ones would facilitate the activity of this nucleus. The nucleus reticularis thalami projects to that thalamic relay nuclei and inhibits thalamic relay to the cortex. ${ }^{6}$ Lesions of the association cortex would result in less inhibition of the ipsilateral nucleus reticularis, in turn leading to an increase in the inhibition of the thalamic sensory nuclei and raising the sensory threshold for contralateral stimuli. During bilateral simultaneous stimulation, the activity of the nucleus reticularis on the damaged side would be further increased by the facilitatory projections from the normal side and cause the extinction of the contralateral stimulus.

Focusing on the temporal rather than on the anatomical determinants of extinction, Birch et al ${ }^{7}$ pronosed that due to its slower processing of information, the damaged hemisphere is more subject to interference from the normal side. They supported this model by demonstrating that stimulating the affected side in advance of stimulating the intact side reduced extinction.

Although the models discussed so far emphasise interhemispheric rivalry during bilateral simultaneous stimulation and ascribe an active inhibitory role to the intact hemisphere over the damaged one, other investigators have implicated defects of attention as the underlying mechanism. ${ }^{8-10}$ However, the attentional hypothesis was criticised by Bender, ${ }^{11}$ who concluded in his monograph on the subject that inattention was not a satisfactory explanation, because directing the patient's attention to the 
affected side did not eliminate extinction. Critchley, ${ }^{12}$ on the other hand, attributed a prominent role to defective attention, and pointed out that many patients with parietal lobe damage who show extinction are in fact unable to direct their attention to the side opposite their lesion. Denny-Brown ${ }^{13}$ made a similar observation in a patient with trimodal extinction after a right parietal infarct who also showed a "persistent deficit in awareness relating to the left side of the body and extrapersonal space". Denny-Brown believed the disorder represented a disturbance in the cortical spatial summation of sensory data and that this "amorphosynthesis" was the result of damage to the "parietal half-center of perception". Although Denny-Brown regarded attention as an epiphenomenon, secondary to the disorder of perception, he did observe that "its application to the left side was transitory".

The role of attention was reemphasised by Heilman, ${ }^{14}$ who proposed the limited-attention theory of extinction. According to this theory, under normal circumstances each hemisphere processes the contralateral stimulus when bilateral simultaneous stimulation is given. Damage to a hemisphere renders the organism inattentive to stimuli on the contralateral side. As the organism recovers, the ability to attend to contralateral stimuli is regained, and this may be mediated by the normal hemisphere. However, the normal hemisphere may have limited attentional capacity. During bilateral simultaneous stimulation, the normal hemisphere's attentional mechanisms are occupied by the contralateral stimulus and are unable to attend to the ipsilateral one. In addition to postulating that extinction may be related to limited attentional capacity, Heilman and coworkers ${ }^{515}$ also suggested that each hemisphere was not only responsible for attending to stimuli in the contralateral visual hemifield (VHF) but also may be attending to stimuli in contralateral head and body hemispace, independent of VHFs.

Hemispace is a complex concept that can be defined according to VHFs, head position, or trunk position. Head and body hemispace refers to the external space to the left or right of the body midline. With the eyes and head facing directly ahead, VHFs and side of head and body hemispace are perfectly aligned. However, under conditions of eccentric fixation, one can dissociate VHF from head and body hemispatial field. For example, if the eyes are directed to the far right, the left VHF will fall within right head and body hemispace.

During traditional testing for extinction to bilateral simultaneous stimulation in the visual modality, a one-to-one relationship exists between the hemispatial field in which the stimuli are presented and the VHF to which they are presented (that is, the stimulus in right hemispace is presented to the right VHF; the $\bar{Z}$ stimulus in left hemispace is presented to the left VHF). Although extinction under these circumstances is compatible with the limited attention theory, it could also be induced by interhemispheric rivalry. Further, because the hemispatial fields are confounded with the VHFs, it is not possible to determine whether extinction depends on the hemispatial location of the stimuli or their retinotopic location, or $\underset{.}{2}$ both.

Under special testing conditions, however, one can examine possible hemispace-visual field interactions in visual extinction. The purpose of this study was two-fold: first, to learn whether interhemispheric on rivalry was necessary to induce extinction and, $\stackrel{\odot}{\perp}$ second, to determine whether the retinotopic or the hemispatial location of the stimuli was the critical factor.

\section{Case report}

A 50 year old right-handed man noted sudden onset of leftsided weakness. He had a past history of myocardial of infarction complicated by peripheral embolisation to the right arm and was on long-term warfarin therapy. At the time of admission, he was awake and oriented, but his affect was flat and he appeared unconcerned about his neurç logical deficit. Language functions were intact, and the patient had normal praxis. He bisected lines to the right $\Phi_{-}$ the midline and failed to cross out lines on the left side of tho $\infty$ page in the line cancellation task. Visual acuity was 20/30 in both eyes. Confrontation testing showed the visual fields to be full, but there was left-sided extinction on bilateral simu taneous stimulation. Extinction on the left could also be demonstrated in the auditory modality. The range of extraocular movements was full. Left central facial weakness was pronounced. The patient had left hemiparesis, with a flaccid and plegic left upper extremity and only minimal movements of the left lower extremity. Deep tendon reflexes were brisker on the left, and the plantar response was extensor on that side. Sensation was diminished to all modalities on the left. Computed tomography showed a right frontoparietal infarct in the distribution of the right middle cerebral artery.

SPECIAL STUDIES OF VISUAL EXTINCTION Experiment 1: Double simultaneous stimulation within the same $V H F$ with central fixation

To prevent binocular viewing of the stimulus presented closer to the midline in conditions $a$ and $b$ of this experiment, a cardboard partition was held in the midsagittal plane between the patient's eyes. Under these circumstances stimuli presented in the right hemispatial field are monocularly viewed by the right eye; those in the left hemispatial field are monocularly viewed by the left eye (figs la and $b$ ). The patient was instructed to look straight ahead and fixate at distance. Under these viewing conditions stimuli presented to the temporal visual field of each eye fall on the nasal portion of the retina, and due to the crossing of these fibres at the optic chiasm will be projected to the contralateral hemisphere. Stimuli presented to the nasal visual 


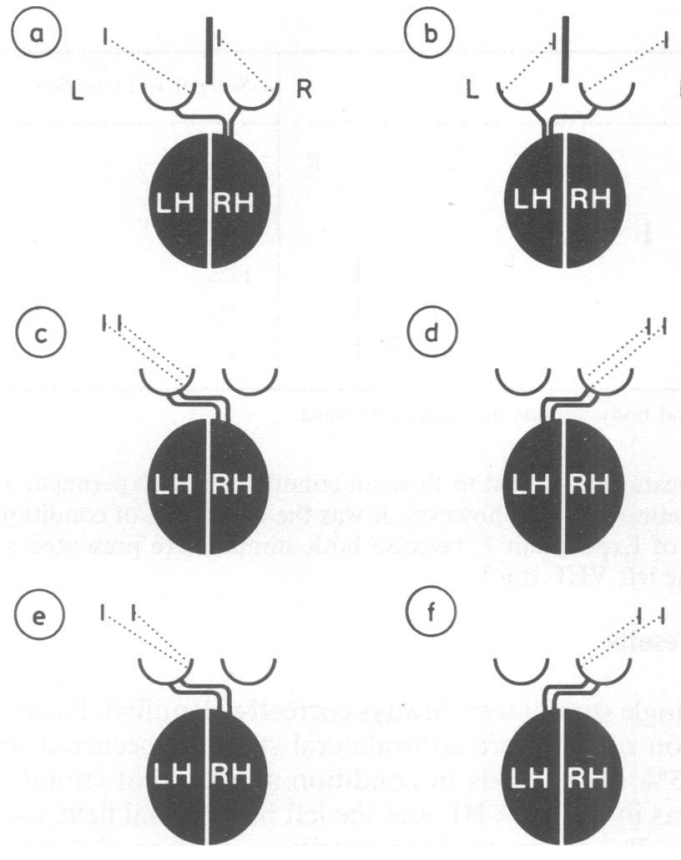

Fig 1 Double simultaneous stimulation within the same $V H F$, with central fixation (experiment 1 ). $L=$ left hemispace; $R=$ right hemispace; $L H=$ left hemisphere; RH $=$ right hemisphere.

field of each eye fall on the temporal portions of the retina and will be projected to the ipsilateral hemisphere by uncrossed temporal fibres.

In conditions $\mathrm{c}, \mathrm{d}, \mathrm{e}$, and $\mathrm{f}$ we tested extinction in the temporal monocular field of each eye and therefore did not use a partition.

Rapid flexions of the examiner's index fingers at the metacarpophalangeal joint served as stimuli and were presented in a frontoparallel plane $10 \mathrm{~cm}$ in front of the patient. After making certain that the patient could detect single stimuli accurately in all sections of the visual fields, double simultaneous stimulation was carried out intermixed with single stimulations in a random order until a total of 30 double simultaneous stimulations were performed in each testing condition. The patient was instructed to say whether he saw the stimulus presented to the left or to the right or whether he saw both in the following testing conditions:

In condition a both stimuli were presented to the left VHF and were projected to the right hemisphere. However, one was in left hemispace and the other was in right hemispace (fig la).

In condition $b$ both stimuli were presented to the right VHF and were projected to the left hemisphere. One was in left hemispace, the other in right hemispace (fig lb).

In condition $c$ both stimuli were in the left hemispace $10 \mathrm{~cm}$ apart and the position of the more peripheral stimulus was the same as in condition a. Both stimuli were presented to the temporal monocular field of the left eye (left VHF), and both were projected to the right hemisphere since they fell on nasal retina (fig lc).

In condition $d$ both stimuli were $10 \mathrm{~cm}$ apart in the right hemispace, and the position of the more peripheral stimulus was the same as in condition b. Both stimuli were presented to the temporal monocular field of the right eye (right VHF), and both were projected to the left hemisphere because they fell on nasal retina (fig ld).

In condition $e$ both stimuli were in the left hemispace and the position of the more peripheral stimulus was the same as in conditions a and $\mathrm{c}$. However, the distance between the stimuli was increased to $20 \mathrm{~cm}$ by presenting the other stimulus $10 \mathrm{~cm}$ closer to the midline than in condition c. Both stimuli were still presented to the temporal monocular field of the left eye (left VHF), and because they fell on nasal retina both were projected to the right hemisphere (fig le).

In condition $f$ both stimuli were in the right hemispace and the position of the more peripheral stimulus was the same as in conditions $\mathrm{b}$ and $\mathrm{d}$. However, the distance between the stimuli was increased to $20 \mathrm{~cm}$ by presenting the other stimulus $10 \mathrm{~cm}$ closer to the midline than in condition $\mathrm{d}$. Both stimuli were still presented to the temporal monocular field of the right eye (right VHF), and were projected to the left hemisphere, since they fell on nasal retina (fig lf).

\section{Results}

Single stimuli were always correctly identified. Of the 30 trials of double simultaneous stimulations within the left VHF, the patient extinguished the stimulus that was more contralateral to the side of the lesion 26 times in condition a $(87 \%), 25$ times in condition c $(83 \%)$, and 24 times in condition e $(80 \%)$. (The term "more contralateral" is used in this paper to designate the stimulus that is on the left side of the pair of stimuli presented simultaneously in all experimental conditions.)

Of the 30 trials of double simultaneous stimulation within the right VHF, extinction of the more contralateral stimulus occurred five times in condition b $(17 \%)$, once in condition $f(3 \%)$, and not at all in condition $d$. The results of Experiment 1 are summarised in table 1.

\section{Discussion}

The results of Experiment 1 demonstrate that extinction can occur during double simultaneous stimulation within the same VHF, thus excluding the possibility that it is caused by interhemispheric rivalry. Instead, there appears to be a limited capacity to attend to the stimulus that is more contralateral to the side of the lesion.

The severity of extinction is determined by the position of the more contralateral stimulus: it is maximal when that stimulus is in the peripheral parts of the left VHF and the left hemispatial field (conditions a, c, and $e$ ), whereas the patient's performance is essentially normal when the more contralateral stimulus is 
Table 1 Summary of results from Experiment 1

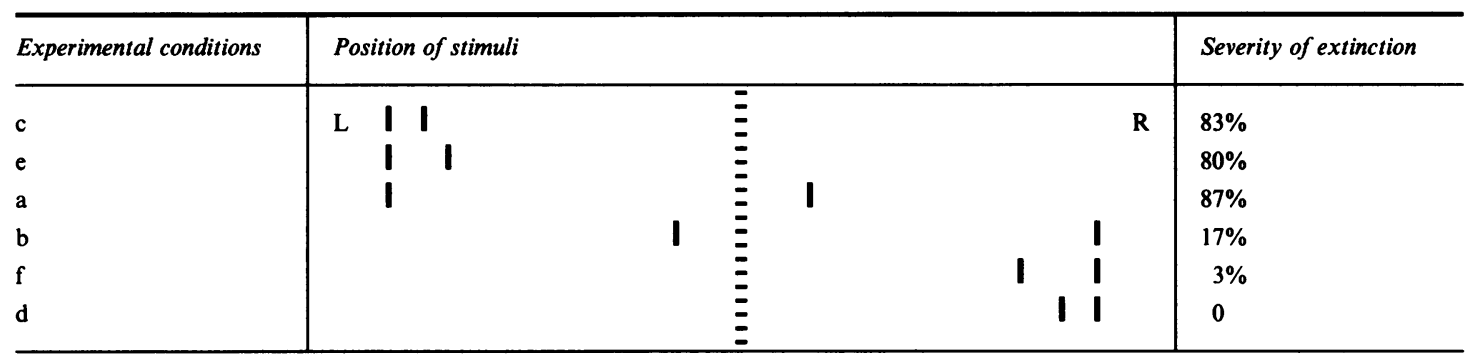

$\mathrm{L}=$ left hemispace; $\mathbf{R}=$ right hemispace. Dotted line represents head and body midline in the sagittal plane.

in the peripheral parts of the right VHF and the right hemispatial field (conditions $d$ and $f$ ). It is also apparent that during double simultaneous stimulation within the right VHF, extinction occurs only when the more contralateral stimulus falls close to the midline within left hemispatial field (condition b).

To learn whether the retinotopic or the hemispatial position of the more contralateral stimulus is the critical factor in determining the severity of extinction during double simultaneous stimulation within the same VHF, we need to test for extinction during eccentric fixation, since under those circumstances the VHFs and head and body hemispatial fields are no longer confounded with each other.

Experiment 2: Double simultaneous stimulation within the same $V H F$ with eccentric fixation.

In the previous experiment we found that the location of the stimulus that is more contralateral to the side of the lesion determines the severity of extinction. In this experiment we attempted to define whether the retinotopic or the hemispatial position of the more contralateral stimulus is the critical factor.

Because this set of experiments was conducted some time after Experiment 1, we first repeated conditions $a$ and $b$ of that experiment to establish a new baseline (figs $2 a$ and $b$ ). We then asked our patient to deviate his eyes $30^{\circ}$ to the left, without changing head or body position. The cardboard partition was also rotated $30^{\circ}$ to the left, so that it was positioned halfway between the new fixation axes of the eyes (fig 2). The patient was again instructed to gaze at distance. The stimuli were then presented at hemispatial positions identical to those of condition a in Experiment 1. However, because of eccentric fixation, these stimuli would now be presented to the right VHF, with the stimulus in left hemispace falling on the temporal retina of the left eye and the stimulus in right hemispace falling on the nasal retina of the right eye. Retinotopically, therefore, this is the equivalent of condition b of Experiment 1, but hemispatially it is identical to condition a of that experiment (fig 2). As before, 30 double simultaneous stimulations were performed intermixed with single stimulations in a random order, and the patient's response mode also remained the same.

The methods used for testing extinction with eccentric fixation to the right were similar to those described above, except that now the stimuli were presented at hemispatial locations identical to those in condition b of Experiment 1. Retinotopically, however, it was the equivalent of condition a of Experiment 1, because both stimuli were presented to the left VHF (fig 2).

\section{Results}

Single stimuli were always correctly identified. Extinction of the more contralateral stimulus occurred on $93 \%$ of the trials in condition a when that stimulus was in the left VHF and the left hemispatial field, and on $7 \%$ of the trials in condition $b$ when the more contralateral stimulus was in the right VHF but in left hemispatial field.

Extinction of the more contralateral stimulus occurred on 14 of the 30 trials of double simultaneouso stimulation with eccentric fixation to the left $(47 \%)$ With eccentric fixation to the right, the patient extinguished the more contralateral stimulus on 23 of the 30 trials of double simultaneous stimulations $(77 \%)$.

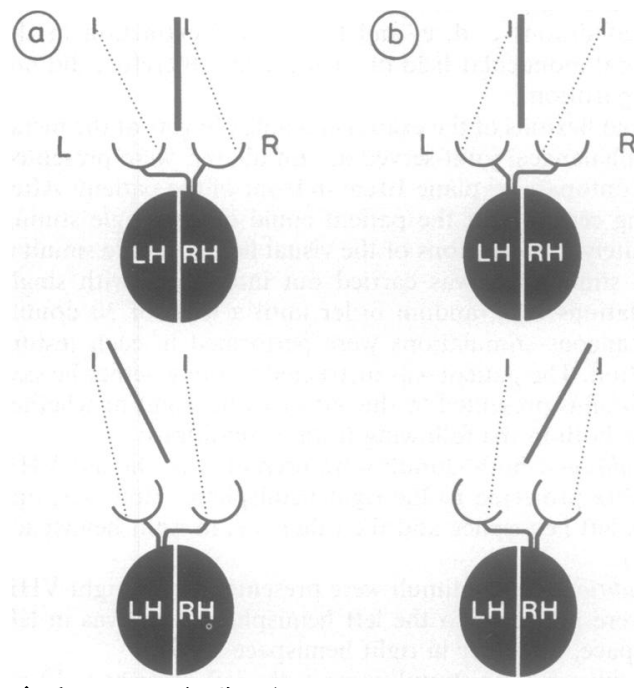

Left eccentric fixation Right eccentric fixation

Fig 2 Double simultaneous stimulation within the same $V H F$, with eccentric fixation (experiment 2 ). 
Table 2 Summary of results from Experiment 2

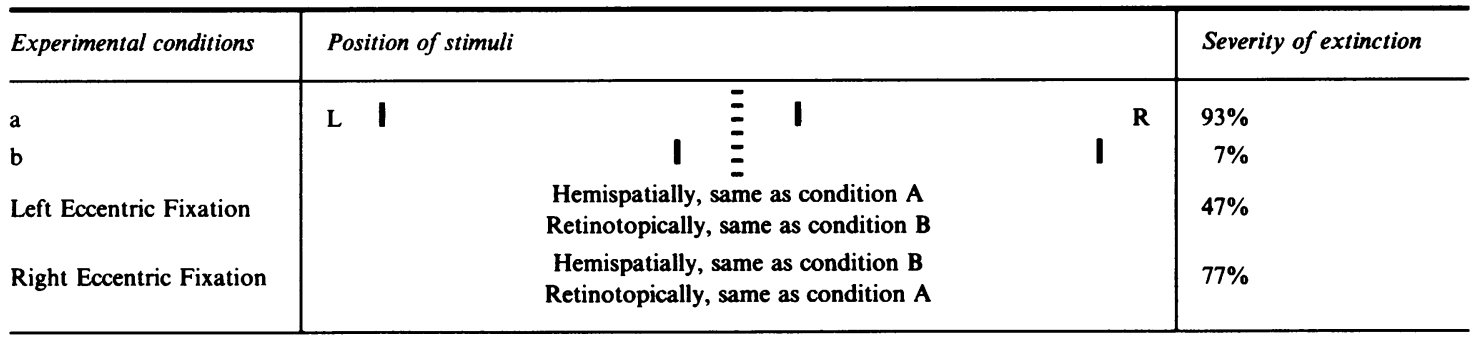

The results of Experiment 2 are summarised in table 2.

\section{Discussion}

As shown by the results of Experiment 2, changes in the angle of gaze during eccentric fixation influenced the severity of extinction under conditions that were hemispatially identical (left eccentric fixation and condition a; right eccentric fixation and condition b), indicating that the retinotopic position of the more contralateral stimulus is a significant factor. However, it is not the only factor, because the severity of extinction was not the same under conditions that were retinotopically identical, but where the hemispatial position of the more contralateral stimulus was different (left eccentric fixation and condition b: right eccentric fixation and condition a). These findings suggest that both the retinotopic and the hemispatial position of the more contralateral stimulus are significant in determining the severity of extinction.

\section{Experiment 3: Double simultaneous stimulation with stimuli in different visual fields.}

Although interhemispheric rivalry cannot account for extinction during double simultaneous stimulation within the same VHF, such rivalry might be involved when the stimuli are presented simultaneously to different VHFs. In this experiment we tried to determine whether interhemispheric rivalry that could occur during double simultaneous stimulation with stimuli in different VHFs alters the pattern of extinction found in Experiment 1 with double simultaneous stimulation within the same VHF.

In this experiment the cardboard partition was used as in conditions $\mathrm{a}$ and $\mathrm{b}$ of Experiment 1 .

In condition $a$ both stimuli were in the left hemispace and were monocularly viewed by the left eye (fig 3a). The stimulus that fell on the nasal retina was projected to the right hemisphere (left VHF), while the stimulus that fell on temporal retina was projected to the left hemisphere (right VHF).

In condition $b$ both stimuli in the right hemispace were monocularly viewed by the right eye (fig $3 b$ ). The stimulus that fell on nasal retina was projected to the left hemisphere (right VHF); the stimulus that fell on temporal retina was projected to the right hemisphere (left VHF).

In condition $c$ the stimulus in the left hemispace was mono- cularly viewed by the left eye, and because the image fell on the nasal retina it was projected to the right hemisphere (left VHF). The stimulus in the right hemispace was monocularly viewed by the right eye, and because it fell on the nasal retina it was projected to the left hemisphere (right VHF). This is the usual clinical method of testing for extinction to double simultaneous stimulation, and if care is taken to ensure that the stimuli are presented to the temporal monocular field of each eye, the use of a partition is not necessary (fig $3 \mathrm{c}$ ).

\section{Results}

Single stimuli were always correctly identified. On $\mathbf{3 0}$ trials of double simultaneous stimulations, the stimulus more contralateral to the lesion was extinguished 28 times in condition a $(93 \%)$, six times in condition b $(20 \%)$ and 27 times in condition c $(90 \%)$. The results of Experiment 3 are summarised in table 3.

\section{Discussion}

Although interhemispheric rivalry is a theoretical possibility during double simultaneous stimulation with stimuli in different VHFs, our results show that such rivalry does not significantly alter the pattern of

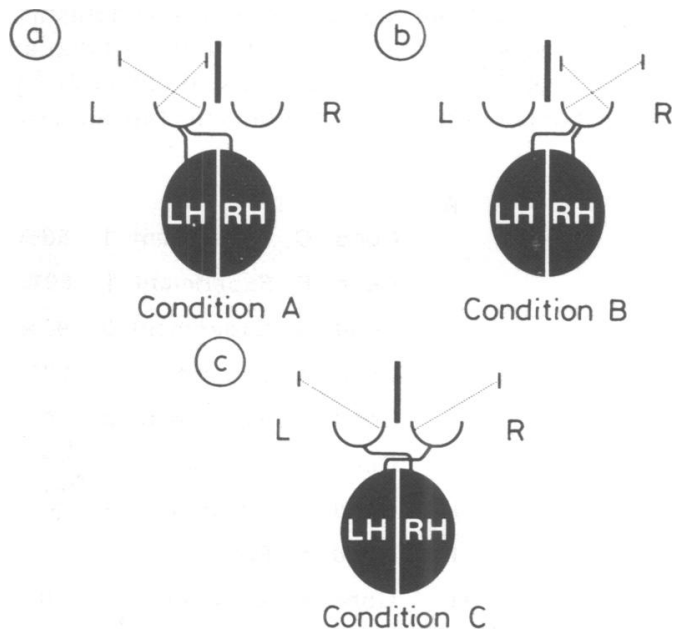

Fig 3 Double simultaneous stimulation with stimuli in different VHFs (experiment 3). 
Table 3 Summary of results from Experiment 3

\begin{tabular}{|c|c|c|c|c|}
\hline Experimental conditions & Position of stimuli & & & Severity of extinction \\
\hline $\begin{array}{l}a \\
b \\
c\end{array}$ & $\begin{array}{ll}\mathrm{L} & \mathbf{1} \\
& \mathbf{I}\end{array}$ & $\begin{aligned} & \equiv \\
& \equiv \\
& \equiv\end{aligned}$ & $\mathbf{I}^{\mathrm{R}}$ & $\begin{array}{l}93 \% \\
20 \% \\
90 \%\end{array}$ \\
\hline
\end{tabular}

extinction found in Experiment 1 with double simultaneous stimulation within the same VHF. Again, extinction was most severe when the more contralateral stimulus falls within the peripheral parts of the left VHF and the left hemispatial field (conditions $a$ and c). Extinction was much less severe when the more contralateral stimulus was close to the midline, although this time in the right hemispatial field (condition $b$ ). The results of Experiments 1 and 3 are summarised in fig 4 . These results suggest that even during double simultaneous stimulation with stimuli in different VHFs, it is the location of the more contralateral stimulus that determines the severity of extinction and also that the location of the other stimulus is not relevant.

\section{General discussion}

When extinction follows damage to the cerebral hemispheres, it occurs most commonly after lesions in the temporoparietal region. ${ }^{16}$ Extinction can be seen in isolation (often in the course of recovery from a more profound hemi-inattention) or with other components of the neglect syndrome, such as hemiakinesia and hemispatial neglect. ${ }^{5}$ It has been proposed that the neglect syndrome is due to a unilateral disturbance of arousal and attentional mechanisms, caused by a dysfunction in a corticolimbic-reticular formation loop. ${ }^{17-19}$ This is manifested clinically by the abnormal performance of patients in contralateral

\begin{tabular}{|c|c|c|c|c|c|c|c|}
\hline $\mathbf{L}$ & & & $\mathbf{R}$ & & & & \\
\hline II & & & & Cond & C, Experiment & 1 & $83 \%$ \\
\hline 11 & & & & Cond & E, Experiment & 1 & $80 \%$ \\
\hline I & $\mathbf{I}$ & & & Cond & A, Experiment & 3 & $93 \%$ \\
\hline I & & I & & Cond & A, Experiment & 1 & $87 \%$ \\
\hline I & & & I & Cond & C, Experiment & 3 & $90 \%$ \\
\hline & $\mathbf{I}$ & & $\mathbf{I}$ & Cond & B, Experiment & 1 & $17 \%$ \\
\hline & & I & $\mathbf{I}$ & Cond & B, Experiment & 3 & $20 \%$ \\
\hline & & I & $\mathbf{I}$ & Cond & F, Experiment & 1 & $3 \%$ \\
\hline & $\vdots$ & & II & Cond & D, Experiment & 1 & $\mathbf{0}$ \\
\hline
\end{tabular}

Fig 4 Summary of results from experiments 1 and 3. $L=$ left hemispace; $R=$ right hemispace. Dotted line represents head and body midline in the sagittal plane. hemispace on a variety of perceptual and motor tasks, pointing to a disturbance of the hemispheric mechanisms that are involved in the perception and the mediation of activities in contralateral hemispace. Evidence from previous studies suggests that head and body hemispace are important in determining the symptoms of hemispatial neglect. ${ }^{2021}$ However, the role of hemispace-visual field interactions in extinction that occurs in the setting of the neglect syndrome has not been previously addressed.

We have demonstrated that extinction occurs during double simultaneous stimulation within the same VHF. Extinction under these circumstances cannot be explained by interhemispheric rivalry, since the stimuli are presented to one VHF only. It seems, rather, that after damage to the right hemisphere there is reduced capacity to attend to the stimulus that is more contralateral to the side of the lesion during double simultaneous stimulation. It is also apparento that extinction is most severe when the more cone tralateral stimulus is in the peripheral parts of lefto VHF or left hemispatial field, while the patient's per formance is essentially normal when the more con tralateral stimulus is in the peripheral parts of right VHF or right hemispatial field. This suggests that inattention to the more contralateral of two simultaneously presented stimuli has a gradient of severity from a maximum in extreme contralateral hemifield or hemispace to a minimum in extreme ipsilateral hemifield or hemispace. However, from the results of Experiment 1 alone we could not determine whether the hemispatial location of the more contralateral stimulus or its retinotopic location was the critical factor.

To answer this question, we attempted to dissociate VHF from the hemispatial field in Experiment 2. If the hemispatial location of the more contralateral stimulus is the critical factor, then one would anticipate no change in the severity of extinction during eccentric fixation, since the hemispatial positions of the stimuli with reference to a head-and-body-centred coordinate system remained unchanged, despite a change of their retinotopic locations (left eccentric fixation and condition a of Experiment 1; right eccentric fixation and condition b of Experiment 1). If, however, the retinotopic location of the more contralateral stimulus is the key determinant, the severity 
of extinction during double simultaneous stimulation will vary with the angle of gaze. A further prediction is that if the retinotopic location of the more contralateral stimulus is the sole determinant, the severity of extinction should be the same in the conditions that are retinotopically identical (left eccentric fixation and condition $b$ of Experiment 1, right eccentric fixation and condition a of Experiment 1), even though the hemispatial locations of the stimuli are different.

The results of Experiment 2 show that the severity of extinction is influenced by the angle of gaze. At first glance this could be interpreted to suggest that the retinotopic location of the more contralateral stimulus is the critical factor. However, it is also apparent that the severity of extinction was not the same in the conditions that were retinotopically identical but hemispatially different $(47 \%$ with left eccentric fixation but $7 \%$ in condition $b ; 77 \%$ with right eccentric fixation but $93 \%$ in condition a), indicating that the hemispatial position of the more contralateral stimulus was also an important factor.

Although interhemispheric rivalry cannot account for extinction that occurs during double simultaneous stimulation within the same VHF, such rivalry could conceivably have an influence on extinction during double simultaneous stimulation within different VHFs (Experiment 3). However, interhemispheric rivalry alone cannot explain why the severity of extinction varied as a function of the position of the more contralateral stimulus (conditions a and $c$ versus condition $b$ in Experiment 3). The results of Experiment 3 are similar to our previous finding of a hemispatial/visual field gradient of inattention to the more contralateral of two simultaneously presented stimuli within the same VHF in Experiment 1. This gradient of inattention to the more contralateral stimulus therefore determines the severity of extinction during double simultaneous stimulation within different VHFs as well.

Zihl and Cramon ${ }^{22}$ studied visual extinction during double simultaneous stimulation within different VHFs in a patient who had a left temporoparietal infarction and had CT scan evidence of an infarction of the left posterior thalamus as well. When double simultaneous stimulation of homologous regions in the left and right VHFs were performed, the severity of the extinction increased as a function of the eccentricity of the stimulus contralateral to the side of the lesion. Extinction of the contralateral stimulus occurred on the majority of the trials beyond $25^{\circ}$, thus suggesting again that there is a gradient of inattention to the more contralateral stimulus that is a function of retinotopic or hemispatial eccentricity.

Invoking interhemispheric rivalry to explain extinction that occurs during double simultaneous stimulation within different VHFs therefore seems superfluous. Based on the results of our experiments, we propose that extinction to double simultaneous stimulation in the setting of the neglect syndrome is due to a reduced capacity to attend to the stimulus that is more contralateral to the side of the lesion. After damage to the right hemisphere, this inattention to the more contralateral stimulus affects the entire unified binocular visual field and spatial attentional field, rather than a particular VHF or hemispatial field, but with a gradient of severity that is determined by the position of the more contralateral stimulus both within a retinotopic coordinate system and in reference to a head-and-body-centred spatial coordinate system. The position of the other stimulus is not relevant (see fig 4).

Studies of single cell recordings in primates offer some clues to the neuronal mechanisms that mediate the function of attending to stimuli within the different sections of visual space. Recording from cells in the postarcuate cortex of the monkey, Rizzolatti et $\mathbf{l}^{\mathbf{2 3}}$ found that of the neurons studied, $29 \%$ had exclusively contralateral visual receptive fields, $2 \%$ had ipsilateral receptive fields, and $69 \%$ had bilateral receptive fields. The bilateral fields were always around the midline, with various lateral extensions according to the neuron, and the responses elicited from the contralateral field were often stronger than the response to ipsilateral stimuli. Rizzolatti et $a^{24}$ postulated that because of this arrangement, the most lateral part of visual space is represented almost exclusively in one hemisphere, whereas the central part of visual space is represented in both hemispheres. After a lesion in one hemisphere, the capacity to orient to visual stimuli will therefore be affected in the entire visual space, but with a gradient of severity from a maximum in extreme contralateral hemifield or hemispace to a minimum in extreme ipsilateral hemifield or hemispace.

In addition to the cell populations in the postarcuate cortex studied by Rizzolatti et al, ${ }^{23}$ single cell recordings from area 7 of the parietal lobes of monkeys have also identified a group of neurons, variously called visual space neurons ${ }^{25}$ or light-sensitive neurons, ${ }^{26}$ that have an important role in the direction of visual attention. Light-sensitive neurons have large contralateral or bilateral receptive fields that usually spare the fovea. ${ }^{27}$ The discharge rate in some of these neurons could also be suppressed by light stimuli in certain areas of their receptive fields. Although the suppressive and excitatory zones were spatially separate, no regular relation between the two was established. ${ }^{27}$ The most intense activity in some of these cells is evoked by stimuli placed most distant from the central line of gaze. ${ }^{26}$ The lightsensitive neurons are probably involved in the attrac- 
tion of gaze and attention toward stimuli in the most peripheral parts of the visual fields, especially within the monocular crescents, and play an important role in mediating the functions of ambient vision. ${ }^{2728}$ The responses of the parietal light-sensitive neurons to visual stimuli are also significantly influenced by the angle of gaze; this finding suggests that the intensity of their response depends both upon the retinotopic location of the visual stimulus and its spatial position. ${ }^{29}$ Andersen and Mountcastle ${ }^{29}$ proposed that there is an interaction between spatial fields mapped by changes in the angle of gaze effect on neuronal responses to visual stimuli and the retinotopic receptive fields and that such a mechanism may function to shape the spatial field of responsiveness of the ambient visual system. Given the response properties of the light-sensitive neurons, damage to these cells from a unilateral parietal lobe lesion could conceivably result in an inability to attend to the more contralateral of two simultaneously presented stimuli with a maximum of severity when the more contralateral stimulus falls within the contralateral monocular crescent or most peripheral part of contralateral hemispace.

Area 7 and the periarcuate cortex are reciprocally connected and are part of the corticolimbic-reticular system that is implicated in mediating attention to stimuli in contralateral hemispace. ${ }^{5}$ As shown by the present case, damage to this system causes extinction during double simultaneous stimulation, due to a limited capacity to attend to the stimulus that is more contralateral to the side of the lesion. Extinction during double simultaneous stimulation in the visual modality therefore represents an inability to distribute the limited attentional resources equally to two different locations. The severity of extinction is a measure of an unequal distribution of attention along a gradient within visual space. The exact neurophysiological correlates of this postulated reduction of attentional capacity, as well as the mechanisms that determine its distribution, however, remain unknown.

This work was supported by the Medical Research Service of the Veterans Administration, National Institutes of Health Grant MH-34752, and the Memory Disorders Clinic, State of Florida.

\section{References}

1 Schwartz AS, Marchok PL, Kreinick CJ, Flynn RE. The asymmetric lateralization of tactile extinction in patients with unilateral cerebral dysfunction. Brain 1979;102:669-84.

2 Nathan PW. On simultaneous bilateral stimulation of the body in a lesion of the parietal lobe. Brain 1946;69:325-34.

3 Reider N. Phenomena of sensory suppression. AMA Arch Neurol Psychiatry 1946;55:583-90.

4 Furmanski AR. The phenomena of sensory suppression. $A M A$ Arch Neurol Psychiatry 1950;63:205-17.

5 Heilman KM, Watson RT, Valenstein E. Neglect and related disorders. In: Heilman KM, Valenstein E, eds. Clinical Neuropsychology, 2nd ed. New York: Oxford University Press, 1985:243-93

6 Scheibel ME, Scheibel AB. The organization of the nucleus reticularis thalami: a golgi study. Brain Res 1966;1:43-62.

7 Birch HG, Belmont I, Karp E. Delayed information processing and extinction following cerebral damage. Brain 1967;90: 113-30.

8 Head H, Holmes G. Sensory disturbances from cerebral lesions. Brain 1911;34:102-254.

9 Poppelreuter WL. Die psychischen Schadigungen durch Kopfschuss im Kriege 1914-1916. Die Störungen der niederen und hoheren Leistungen durch Verletzungen des Oksipitalhirns. vol 1. Leipzig: Leopold Voss. 1917. Referred to by M Critchley. Brain 1949;72:540.

10 Allen IM. Unilateral visual inattention. $N Z$ Med $J$ 1948;47: 605-17.

11 Bender MB. Disorders of Perception. Springfield, Ill: Charles C Thomas, 1952.

12 Critchley $M$. The phenomenon of tactile inattention with special reference to parietal lesions. Brain 1949;72:538-61.

13 Denny-Brown D, Meyer JS, Horenstein S. The significance of perceptual rivalry resulting from parietal lesions. Brain 1952;75:433-71.

14 Heilman KM. Neglect and related disorders. In: Heilman KM Valenstein E, eds. Clinical Neuropsychology. New York: Oxford University Press, 1979:268-307.

15 Bowers D, Heilman KM, Van Den Abell T. Hemispace-visual field compatibility. Neuropsychologia 1981;19:757-65.

16 Heilman KM, Watson RT, Valenstein E, Damasio AR. Localization of neglect. In: Kertesz A, ed. Localization in Neuropsychology. New York: Academic Press, 1983:471-92.

17 Heilman KM, Valenstein E. Frontal lobe neglect in man. Neuro $\log y$ 1972;22:660-4.

18 Watson RT, Heilman KM, Cauthen JC, King FA. Neglect afte cingulectomy. Neurology 1973;23:1003-7.

19 Watson RT, Valenstein E, Heilman KM. Thalamic neglect: poss ible role of the medial thalamus and nucleus reticularis thalam in behavior. Arch Neurol 1981;38:501-6.

20 Heilman KM, Valenstein E. Mechanisms underlying hemispatia neglect. Ann Neurol 1979;5:166-70.

21 Bowers D, Heilman KM. Pseudoneglect: effects of hemispace on tactile line bisection task. Neuropsychologia 1980;18:491-8.

22 Zihl J, Cramon D von. The contribution of the 'second' visual system to directed visual attention in man. Brain 1979;102: 835-56.

23 Rizzolatti G, Scandolara C, Matelli M, Gentilucci M. Afferent properties of periarcuate neurons in macaque monkeys. II. Visual responses. Behav Brain Res 1981;2:147-63.

24 Rizzolatti G, Gentilucci M, Matelli M. Selective spatial attention: one center, one circuit or many circuits? In: Posner MI, Marin OS, eds. Attention and Performance XI. Hillsdale, New Jersey: Lawrence Erlbaum Associates, 1985:251-69.

25 Mountcastle VB, Lynch FC, Georgopoulos A, Sakata H, Acuna C. Posterior parietal association cortex of the monkey: command functions for operations within extrapersonal space. J Neurophysiol 1975;38:871-908.

26 Yin TCT, Mountcastle VB. Visual input to the visuomotor mechanisms of the monkey's parietal lobe. Science 1977;197:1381-3.

27 Motter BC, Mountcastle VB. The functional properties of the light sensitive neurons of the posterior parietal cortex studied in waking monkeys: foveal sparing and opponent vector orientation. J Neurosci 1981;1:3-26.

28 Mountcastle VB, Anderson RA, Motter BC. The influence of attentive fixation upon the excitability of the light sensitive neurons of the posterior parietal cortex. J Neurosci 1981;1:1218-35.

29 Andersen RA, Mountcastle VB. The influence of the angle of gaze upon the excitability of the light sensitive neurons of the posterior parietal cortex. $J$ Neurosci 1983;3:532-48. 